\title{
Applications of efficiency and productivity analysis: editors' introduction
}

\author{
Subal C. Kumbhakar $\Upsilon^{1} \cdot$ Emir Malikov $\Upsilon^{2} \cdot$ Christopher F. Parmeter $\Upsilon^{3}$ \\ Received: 12 April 2021 / Accepted: 13 April 2021 / Published online: 3 May 2021 \\ (c) The Author(s), under exclusive licence to Springer-Verlag GmbH Germany, part of Springer Nature 2021
}

This special issue collects research from many of the leaders in both the application and the theory of productivity and inefficiency. We started this special issue prior to the COVID-19 pandemic, and with individuals and firm shaving to adapt on the fly to lockdowns, social distancing, masking and the perils of operating in a nonstandard environment, both understanding the presence of (in)efficiency and how to improve productivity have taken on heightened importance.

Firm-level technology and productivity are key inputs to models in many fields including trade, labor, environmental economics, industrial organization, financial economics as well as management, rendering the measurement of efficiency and productivity impact across many different areas beyond just microeconomic analysis of the firm. This field is constantly evolving as new approaches are developed and implemented on a near weekly basis. For evidence of this look, no further than the biannual conferences run in Europe, North America and the Pacific Rim which routinely host myriad "methods" and "applications" sessions where leading scholars discuss cutting-edge techniques and their empirical applications targeted at solving outstanding issues across many fields of economics, operation research, engineering, etc.

Our aim with this special issue of Empirical Economics is to bring together contributions both from the preeminent experts actively working on the development of new and robust methodologies for the measurement of efficiency/productivity as well as

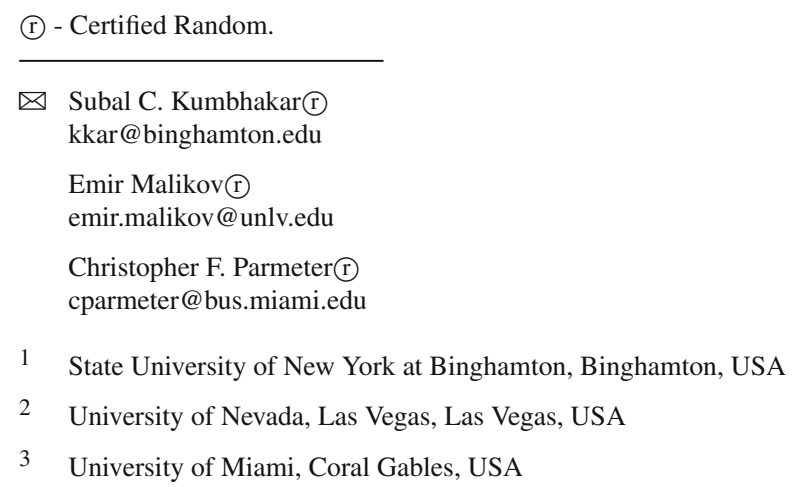


from many leading applied researchers whose work focuses on the innovative empirical implementation of said methodologies. The underlying idea is to provide practitioners with a select collection of papers showcasing the latest developments in the field across various empirical contexts in order to illustrate their practical capabilities and versatility.

We sought to embrace the research articles focused on the original application of different paradigms in the measurement of productivity and efficiency-including stochastic frontier, data envelopment analysis as well as proxy-based approachesto construct a special issue that highlights the wide array of application domains, consistent with the aims and scopes of the journal. This special issue is a testament to the strength and vibrancy of the field. Contributed empirical applications range from energy, banking, agricultural production, manufacturing, health care and hospitality sector, utilities, education and the environment.

The call for papers was widely publicized, and we were happy to receive many highquality submissions. All papers submitted for the special issue have gone through the standard review process of the journal. In the end, eighteen papers were accepted for publication in this special issue, and they feature both solicited and unsolicited contributions.

Before we provide our own interpretation and context to the papers that appear in the special issues we would like to point out to our readers that it is worth their time to go through the full special issue. All of the papers here have important insights and novel applications. Additionally, many of the themes and concepts in this special issue cut across various application domains and have broad appeal to the economics profession. The papers are thematically different but unified in their focus on issues pertaining to efficiency and productivity. We believe that these articles will stand the test of time and should serve as beacons for various issues in efficiency and productivity for years to come. There is the natural delineation of papers using stochastic frontier analysis and those using data envelopment analysis. But these are not simple rote applications. Many have new and interesting datasets (Hazarika and Paul 2020; Argüelles and Orea 2020; O'Loughlin and Wilson 2021; Andor et al. 2020; Mosheim and Sickles 2021). Nearly all of the papers that appear here put forth major econometric and economic advances for these well-worn models and in a variety of subdisciplines of econometrics: nonparametric, spatial, Bayesian, index numbers, etc.

We owe a debt of gratitude to the contributing authors for trusting us to handle their research at the journal rather than submit elsewhere and for our many pleas to stay on top of revisions. The strength of any journal is the authors who submit their research to it. On a final note, we would like to thank the many very capable referees who provided excellent criticism and feedback on the contributed papers. We thank them for their dedicated service in helping us put this special issue together and ensuring its high quality. We also owe a great debt of appreciation to the editorial board of Empirical Economics for approving this issue in the first place.

A brief overview of the included contributions in the order of their publication, which was determined chiefly based on their methodological and thematic clustering, follows. 
The special issue opens with a series of innovative applications of the methods for identifying frontiers and measuring efficiency in unconventional contexts.

In a pursuit of an explanation to the "calorie consumption puzzle," Hazarika and Paul (2020) apply the stochastic cost frontier methods to household data on calorie consumption expenditures in India. With this new way of gauging temporal changes in food security, they find that Indian households' purchases of calories became more expenditure-efficient over time at all income levels, suggesting that there was a squeeze in the household food budget which helps explain the emergence of the "puzzle" whereby the per capita calorie consumption declined despite rising average incomes in the country.

Motivated by the popular use of "output gap" measures to predict recessions, Mastromarco et al. (2021) show how (latent) technical inefficiency from the standard production theory can be utilized to quantify such a gap between the potential and actual outputs for countries. Using this new frontier-based measure of country's output gap as a key predictor, they then estimate probability of an economic recession in Italy using the generalized nonparametric quasi-likelihood dynamic discrete choice model.

Argüelles and Orea (2020) fuse the unique bottom-up spatial model with a stochastic frontier model to examine external and internal power supply interruptions in Spanish municipalities. The particularly notable feature of their analysis is that their spatial model differs from the conventional specifications in that it is developed from the bottom up using the information on each individual power supply interruption and the clear directionality of the chain reaction of interruptions that followed. This enables them to properly model contagion, diffusion and spillover effects across municipalities that are physically connected through the distribution network.

Using a well-known two-tier stochastic frontier model, Papadopoulos (2020) takes on a task of measuring the effect of firm management on output when the data on management as a production factor is unavailable. Treating management as a latent variable, he develops a production frontier model that is consistent with the theory of firm and can be used to identify the effect of management on the firm output separately from inefficiency. With the use of the World Management Survey data to validate his novel approach, Papadopoulos (2020) finds that the estimates from his latent-variable model align well with those obtained using the observable measures of management, when such are indeed available.

Other contributions to the special issue focused more on methodological extensions and generalizations of the work-horse models for the efficiency and productivity measurement.

To measure efficiency of different groups of hospitals (teaching and non-teaching) in Queensland, Australia, Nguyen and Zelenyuk (2021) generalize the most recent developments in statistical analysis of aggregate efficiency by extending the central limit theorems for the envelopment estimators to the context where there are several sub-groups in the population.

Also using the envelopment estimator, Ray et al. (2020) focus on the less frequently used geometric Young index to measure productivity change in the Indian manufacturing. They reformulate the standard geometric distance function by removing the restrictions on the individual input and output scaling factors to obtain the benchmark 
input-output bundle on the frontier. In this reformulated model, the geometric Young index is then exhaustively decomposed into the efficiency change and technical change factors even under variable returns to scale. Notably, Ray et al. (2020) show that, in the unrestricted model that they put forward, there is no scale contributor to the geometric Young productivity growth index even when one allows for variable returns to scale.

In light of the growing interest in studying productivity spillovers, Zhao et al. (2020) extend proxy-variable estimators of nonparametric production functions to the case when technology spillovers and common shocks have persistent effects on firm productivity and are controlled for through spatial networks and a factor structure in the productivity evolution process. In doing so, they synthesize the proxy-variable methods of structural identification of production functions with the semiparametric common correlated effect models. The proposed model is then applied to the Chinese computer equipment manufacturing data.

Lai (2020) focuses on extending single-equation stochastic frontier models to the case of multiple equations. Using copulas, she develops the maximum simulated likelihood estimator for a seemingly unrelated system of multiple stochastic frontier equations with random effects and correlated composite errors. She shows that, as expected, the separate estimation of each equation ignores the valuable information contained in the error correlation across frontiers which leads to significant efficiency losses in the estimation of efficiency. Lai (2020) demonstrates the usefulness of her approach by estimating technical efficiency of the international hotels in Taiwan.

Concerned with the endogeneity in the estimation of semi/nonparametric stochastic frontiers and inefficiency, Prokhorov et al. (2020) discuss three estimation strategies for tackling endogenous regressors while imposing theoretic shape restrictions into the frontier. Considered include both the limited information maximum likelihood and the generalized method of moments estimators. To showcase their methodologies, Prokhorov et al. (2020) apply them to the US commercial banking data.

The next two papers take to heart the computational aspects of measuring technical inefficiency in practice using the go-to normal-half-normal stochastic frontier estimators.

Amsler et al. (2020) consider various methods of evaluating the cdf of the skewnormal distribution, which arises in the stochastic frontier models because it is the distribution of the composite error that is the sum (or difference) of a normal and a half-normal random variable. They investigate the accuracy of the evaluation of the cdf using expressions based on the bivariate normal distribution as well as using simulation methods and some approximations. By a simulated example, Amsler et al. (2020) show that the use of approximations instead of the theoretical exact expressions may be critical in obtaining meaningful and valid estimation results.

The composite error in stochastic frontier is routinely specified to have the distribution be skewed in one direction. In practice, however, estimated residuals may display skewness in the wrong direction, in which case model re-specification or pulling a new sample is oftentimes prescribed. Since wrong skewness may manifest as a finite sample problem, Cai et al. (2021) propose a new solution using a finite sample adjustment to the existing estimators to obtain the desired direction of residual skewness. 
This provides an alternative empirical approach to dealing with the wrong skewness problem that does not require model re-specification.

The special issue then concludes with a flurry of papers pursing efficiency/ productivity analyses in a wide range of empirical contexts which illustrates both the breadth and depth of the productivity literature.

Using the nonparametric free disposal hull estimator, O'Loughlin and Wilson (2021) examine the performance of US municipal governments prior to, during, and following the 2008 financial crisis. Their results strongly suggest non-convexity of the local governments' production set, calling into question the results of previous studies examining municipal efficiency using the data envelopment estimators that do not allow for non-convexity.

Motivated by the global efforts at combating climate change, Andor et al. (2020) analyze the energy saving potentials in the residential sector using the unique disaggregated household data from Germany. The authors estimate inefficiency in the residential use of electricity and its determinants via the stochastic input requirement function and find compelling evidence of a notable potential for energy savings.

Feng and Wang (2020) investigate the determinants of the poor profitability of community banks relative to that of large banks in the USA. To do so, they decompose the relative profitability of community banks into five cost-frontier-based explanatory factors, which they estimate using a Bayesian true fixed-effects stochastic cost frontier model. According to their findings, the lower profitability of community banks in 2001-2017 was attributable mainly to their lower technical change, lower scale efficiency and higher funding costs.

Using a four-component stochastic frontier model, Badunenko et al. (2020) evaluate the inefficiency of adult education programs in Belgium. Their main focus is on distinguishing between persistent and transient inefficiency because, whereas the former is structural and difficult to tackle, the latter can be eliminated somewhat easily without major structural changes. Their results indicate that, despite the presence of persistent inefficiency, the overall inefficiency is mainly driven by the transient component, and hence, at the control of the adult education management.

Mosheim and Sickles (2021) focus is on the nexus of agriculture and the environment. In their paper, they explores spatial effects of nitrogen and phosphorus pollution and drinking water production patterns in agriculture. They analyze nutrient pollution based on data collected via the Safe Drinking Water Act of 1974 to estimate how water bodies and sub-basins impaired by nutrient pollution spillover spatially to affect drinking water utilities based on input distance functions that address varying productive efficiencies of water utilities as well as their scale and scope of operations.

Using their recently developed methodology for unbundling technology and total factor productivity (TFP) by estimating technology-specific production function parameters, Battisti et al. (2020) ask whether the productivity advantage of internationalized firms documented in the international trade literature can be interpreted most accurately in terms of proximity to the "technological frontier" or pure productivity premiums. Exploiting detailed data for a sample of firms across various West European countries, they find that technology gaps (with respect to the frontier) are more than three times larger than the TFP gaps, on average. 
The last, but by no means the least important, paper in the issue is Barauskaite and Nguyen (2021) investigation of the direct and inter-sectoral network effects of idiosyncratic TFP shocks on sectors' growth in US manufacturing. To deal with the potential endogeneity of TFP, Barauskaite and Nguyen (2021) propose a novel set of instruments constructed using the technology shocks identified via sign restrictions from the sectoral structural vector autoregression models. Using US input-output tables and industry-level data, they quantify direct and network-based effects of the shocks and find that a positive idiosyncratic technology shock induces large positive direct and network effects on sectoral growth, with the latter spillover effects propagating the shocks mostly downstream.

On a final note, we would like to thank the many very capable referees who provided excellent criticism and feedback on the contributed papers. We thank them for their dedicated service in helping us put this special issue together and ensuring its high quality. We also owe a great debt of appreciation to the editorial board of Empirical Economics for approving this issue in the first place.

\section{References}

Amsler C, Papadopoulos A, Schmidt P (2020) Evaluating the CDF of the skew normal distribution. Empir Econ. https://doi.org/10.1007/s00181-020-01868-6

Andor MA, Bernstein DH, Sommer S (2020) Determining the efficiency of residential electricity consumption. Empir Econ. https://doi.org/10.1007/s00181-020-01967-4

Argüelles P, Orea L (2020) Managing power supply interruptions: a bottom-up spatial (frontier) model with an application to a Spanish electricity network. Empir Econ. https://doi.org/10.1007/s00181020-01968-3

Badunenko O, Mazrekaj D, Kumbhakar SC, De Witte K (2020) Persistent and transient inefficiency in adult education. Empir Econ. https://doi.org/10.1007/s00181-020-01966-5

Barauskaite K, Nguyen ADM (2021) Direct and network effects of idiosyncratic TFP shocks. Empir Econ. https://doi.org/10.1007/s00181-020-02009-9

Battisti M, Belloc F, Del Gatto M (2020) Is the productivity premium of internationalized firms technologydriven?. Empir Econ. https://doi.org/10.1007/s00181-020-01936-x

Cai J, Feng Q, Horrace WC, Wu GL (2021) Wrong skewness and finite sample correction in the normal-half normal stochastic frontier model. Empir Econ. https://doi.org/10.1007/s00181-020-01988-z

Feng G, Wang C (2020) Determinants of profitability of community banks in the U.S.: a cost frontier based decomposition approach. Empir Econ. https://doi.org/10.1007/s00181-020-01952-X

Hazarika G, Paul SB (2020) India's calorie consumption puzzle: insights from the stochastic cost frontier analysis of calorie purchases. Empir Econ. https://doi.org/10.1007/s00181-020-01945-w

Lai H (2020) Maximum simulated likelihood estimation of the seemingly unrelated stochastic frontier regressions. Empir Econ. https://doi.org/10.1007/s00181-020-01962-9

Mastromarco C, Simar L, Zelenyuk V (2021) Predicting recessions with a frontier measure of output gap: an application to Italian economy. Empir Econ. https://doi.org/10.1007/s00181-021-02029-z

Mosheim R, Sickles RC (2021) Spatial effects of nutrient pollution on drinking water production. Empir Econ. https://doi.org/10.1007/s00181-021-02019-1

Nguyen BH, Zelenyuk V (2021) Aggregate efficiency of industry and its groups: the case of Queensland public hospitals. Empir Econ. https://doi.org/10.1007/s00181-020-01994-1

O’Loughlin CT, Wilson PW (2021) Benchmarking the performance of U.S. municipalities. Empir Econ. https://doi.org/10.1007/s00181-021-02026-2

Papadopoulos A (2020) Measuring the effect of management on production: a two-tier stochastic frontier approach. Empir Econ. https://doi.org/10.1007/s00181-020-01946-9

Prokhorov A, Tran KC, Tsionas MG (2020) Estimation of semi- and nonparametric stochastic frontier models with endogenous regressors. Empir Econ. https://doi.org/10.1007/s00181-020-01941-0 
Ray SC, Deb AK, Mukherjee K (2020) Unrestricted geometric distance functions and the geometric Young productivity index: An analysis of Indian manufacturing. Empir Econ. https://doi.org/10.1007/s00181020-01925-0

Zhao S, Man J, Kumbhakar SC (2020) Estimation of firm productivity in the presence of spillovers and common shocks. Empir Econ. https://doi.org/10.1007/s00181-020-01922-3

Publisher's Note Springer Nature remains neutral with regard to jurisdictional claims in published maps and institutional affiliations. 